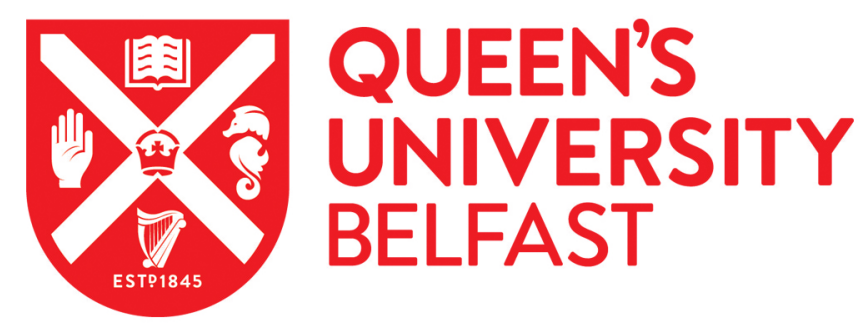

\title{
Rigorous Specification and Low-Latency Implementation of Technical Market Indicators
}

\author{
Bakanov, K., Spence, I., Vandierendonck, H., \& Gillan, C. J. (2014). Rigorous Specification and Low-Latency \\ Implementation of Technical Market Indicators. Paper presented at Parallel Programming for Analytics \\ Applications 2014, Orlando, United States. https://doi.org/10.1145/2567634.2567636
}

\section{Document Version:}

Early version, also known as pre-print

\section{Queen's University Belfast - Research Portal:}

Link to publication record in Queen's University Belfast Research Portal

\section{Publisher rights}

(ङ) ACM, 2014. This is the author's version of the work. It is posted here by permission of ACM for your personal use. Not for redistribution. The definitive version was published in PPAA '14 Proceedings of the first workshop on Parallel programming for analytics applications Pages 43-52\} http://doi.acm.org/10.1145/2567634.2567636"

\section{General rights}

Copyright for the publications made accessible via the Queen's University Belfast Research Portal is retained by the author(s) and / or other copyright owners and it is a condition of accessing these publications that users recognise and abide by the legal requirements associated with these rights.

Take down policy

The Research Portal is Queen's institutional repository that provides access to Queen's research output. Every effort has been made to ensure that content in the Research Portal does not infringe any person's rights, or applicable UK laws. If you discover content in the Research Portal that you believe breaches copyright or violates any law, please contact openaccess@qub.ac.uk. 


\section{Rigorous Specification and Low-Latency Implementation of Technical Market Indicators}

\author{
Konstantin Bakanov Ivor Spence \\ Hans Vandierendonck \\ Queen's University of Belfast \\ kbakanov01@qub.ac.uk i.spence@qub.ac.uk \\ h.vandierendonck@qub.ac.uk
}

\author{
Charles Gillan \\ charlesgillan@handhold.eu
}

\begin{abstract}
Technical market indicators are tools used by technical analysts to understand trends in trading markets. Technical (market) indicators are often calculated in real-time, as trading progresses. This paper presents a mathematicallyfounded framework for calculating technical indicators. Our framework consists of a domain specific language for the unambiguous specification of technical indicators, and a runtime system based on Click, for computing the indicators. We argue that our solution enhances the ease of programming due to aligning our domain-specific language to the mathematical description of technical indicators, and that it enables executing programs in kernel space for decreased latency, without exposing the system to users' programming errors.
\end{abstract}

Keywords domain-specific languages, technical market indicators, in-kernel processing, click router

\section{Introduction}

Technical Analysis is concerned with analyzing stock price movements, identifying patterns in them and then taking advantage of these patterns by making preemptive trades [12]. Traders predominantly relying on technical analysis to conduct their trading are sometimes called "technicians" or "technical analysts". There are many tools which technicians use to first describe and later identify the patterns in stock movements. These range from charting, where traders visually identify patterns in charts, technical market indicators, which usually consist of some mathematical formula encompassing a sliding window view of the market, to more complex tools, such as neural networks or a combination of neural networks and technical market indicators [20, 21].

A technical market indicator is a mathematical formula applied to time series data, which usually consists of high, low, close prices and volume for a given period of time. Conventionally this period of time used to be a trading day, however in the modern age with the rise of day traders, the period of time can be anything from a few seconds to a few

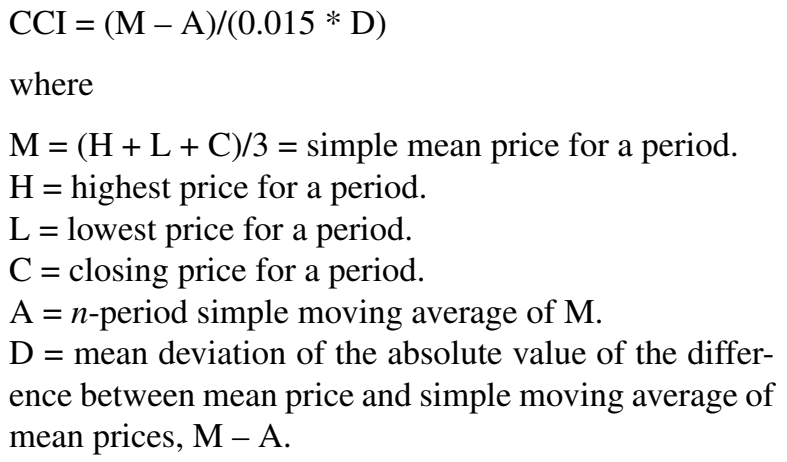

Figure 1. Definition of the Commodity Channel Index [7] (quoted verbatim).

hours $[14,23]$. The technical indicators are therefore used to identify trends within markets and their associated characteristics, such as momentum, sentiment and other properties [8]. Some indicators are used to gauge an entire market, such as Coppock Curve and Advance-Decline Line.

A system to compute technical market indicators in realtime has to combine various properties. It must allow technicians to fairly easily specify the indicators, the specification of the indicators must be rigorous and they must be executed as fast as possible. These are significant challenges, but ones that are well addressed by domain specific languages (DSLs). In this paper, we develop the basis for a domain specific language for specifying and computing technical market indicators.

The specification of technical market indicators is nontrivial. Figure 1 lists the Commodity Channel Index, a price momentum indicator. Clearly, such a specification is not only hard to read, but it is also imprecise. To counter these issues, the specification comes with a detailed computational recipe [7], resembling an algorithm written in an imperative programming style. We have found that there is generally no uniformity of notations. This may lead to ambiguity in interpreting indicators as our discussion on the definition of 
time intervals will show. This work aims to solve these issues by presenting a mathematically founded framework for the definition of technical indicators.

In order to make the specification of technical indicators rigorous, we base our DSL on the mathematical specification of technical market indicators, as elaborated in Section 2. In essence, a technical indicator operates over a sequence of timestamped values (trades), which are summarized into high, low, open and close prices over specific time intervals. Technical indicators are then functions over sequences of high, low, open and close prices. Our DSL will be closely aligned to these mathematical definitions, closing the semantic gap between the specification (mathematics) and the programming language. ${ }^{1}$ Moreover, our formulation is modular, as different operations may be defined individually and reused at will. As an example, we define the exponential weighted moving average (EWMA) as a reusable component in Section 3. This way, we can build up a library of commonly used computations in technical market indicators, which simplifies the programming task.

We apply our framework to the formulation of the directional movement index (DMI), a non-trivial technical market indicator [9]. Section 4 shows how we can specify DMI in a few steps by reusing definitions of high, low, open and close prices as well as EWMA.

We select the Click modular router [15] as a runtime system for our DSL. As such, it is invisible to the programmer and other runtime systems may be selected or designed. Click is, however, particularly appropriate as it is designed to react to incoming (network) packets (in our case trades) and process packets as they arrive. The mapping of functional components in our DSL to Click components is quite straightforward and is well suited to apply code optimization strategies to specialize the Click components to the technical indicators computed. Moreover, the Click router is capable of working with raw market data, coming directly from the exchange (known as a market data feed), thus making it possible to use it as part of a low latency system, which can be appealing to high frequency traders.

As the latency of calculating technical market indicators can be extremely important in algorithmic trading, we demonstrate the use of our DSL to perform the computation of indicators in kernel space (Section 5). Executing programs in kernel space is extremely dangerous, as any software bug may affect the whole system. However, by using a DSL, where the programming language is restricted and where memory accesses are under full control of the DSL compiler and runtime system, we can provide a safe system to execute programs in kernel space. This way, we demonstrate improved processing latency/throughput for the DMI indicator when evaluating the indicator in kernel space.

\footnotetext{
${ }^{1}$ We do not yet define a DSL in this paper, as we are still exploring the principles and appropriate concepts to use, yet the mathematical description and DSL are in direct correspondence.
}

Finally, Section 6 concludes this paper and discusses future work.

\section{The Fundamentals of Technical Indicators}

The basic building blocks of a technical indicator are the following properties: High Price, Low Price, Closing Price, Opening Price (rarely) and Volume. Any one of these characteristics is calculated for a given time interval. Once those values are calculated for every interval in question, then any technical indicator can be represented as a rigorous mathematical formula.

Some indicators are simple and can be recorded with one expression, as in the previous CCI example. Some formulas are more complex and are better recorded as a cascade of functions.

However before moving onto the more complex constructs let us consider first the most basic building block of any technical indicator - that is the time-price interval. For this purpose let us define the prices, the volumes and the times at which these trades have occurred as a sequence of 3 -tuples. Let us name this sequence $D$. In order to select any one member of a given tuple $d$, let us define 3 helper functions:

time $(d)$ - which returns the time component of a tuple $d$. price $(d)$ - which returns the price component of a tuple $d$. volume $(d)$ - which returns the volume component of a tuple $d$.

Let us also assume that the observations started at time 0 .

Now we need to select values $d$ from the sequence $D$, which fall inside a given interval. We need this to calculate High, Low, Close, Open, as these are defined per a given interval. However we have a number of choices in this. Firstly let us name the length of interval $a$, let us name the current time $T$ and let us number the intervals. We'll use variable $i$ for this purpose, so that the first interval would be $i_{0}$, the second interval would be $i_{1}$, the one after $i_{2}$ and so on. The number of current interval $k$ can be calculated as $k=\lfloor T / a\rfloor$. Then the main question can be formulated as this: how do we select values $d$ (for calculating High, Low, Close, Open) when time $T$ occurs inside an interval $i_{k}$ ? There are 3 options here:

1. We only calculate High, Low, Close, Open after an interval has elapsed. I.e. during an interval $i_{k}$ the most recent High, Low, Close, Open would be the ones calculated for the previous interval $i_{k-1}$. Therefore if a technical indicator operates on High, Low, Close, Open from, say, 3 last

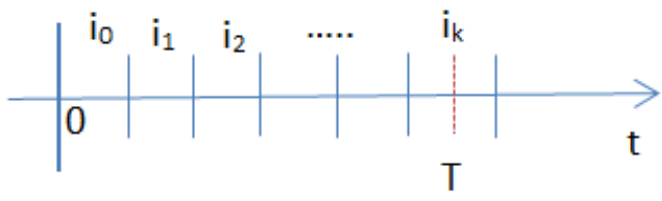

Figure 2. Selection of time intervals under options 1 and 2. Intervals are fixed and are all of equal length. 


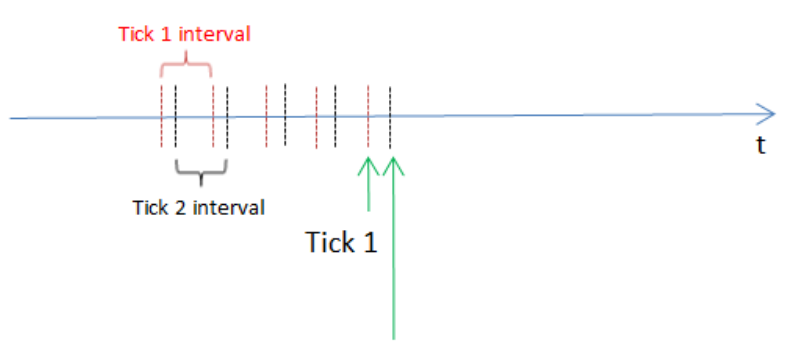

Tick 2

Figure 3. Selection of time intervals under option 3. When calculating indicators in response to the trade occurring at tick 2 the intervals are shifted slightly to more recent trades compared to when calculating indicators in response to tick 1 .

intervals, then these intervals will be $i_{k-1}, i_{k-2}, i_{k-3}$. The figure 2 illustrates the time intervals.

2. Alternatively at the start of the interval $i_{k}$ we set High, Low, Close, Open to that of the interval $i_{k-1}$. Then as prices come in during the current interval $i_{k}$ we update High, Low, Close, Open. In this case the very same indicator as in the option \#1 operating on 3 last intervals will use trades occurring in the intervals $i_{k}, i_{k-1}, i_{k-2}$.

3 . In contrast with the first two options we now consider the case when intervals' boundaries are not set, but rather move with every trade. I.e. the time $T$ will always coincide with the end of interval $i_{k}$. See figure 3 for an illustration of this.

We must also consider the cases when the user at time $T$ wishes to retrieve the technical indicator value at time $T-b$, where $b$ is a random time interval. For the purpose of this discussion we will lay the responsibility for recording previous indicator values upon the user. I.e., in this paper we focus on real-time and leave historical values as future work. Also it is important to note that our point of reference is current interval $k$ and all other intervals are defined as an offset $i$ from $k$.

With the above points in mind let us mathematically formulate the selection of time intervals from a relation $D$. The selection can be done using sequence comprehension. Let us name the function, which does the selection, an $F$ - for filter.

For the option \#1 the formulas will look as follows:

$$
\begin{aligned}
F_{1}(D, T, i, a)= & {[d: D \mid(k-i) * a \leq t i m e(d)<} \\
& (k-i+1) * a \wedge(k-i) \geq 0]
\end{aligned}
$$

where

$d$ is a dummy variable.

$T$ is the current time.

$a$ is the length of the interval in question.

$k$ is the number of the current interval, calculated as $k=\lfloor T / a\rfloor$.

$i$ is the offset of the interval in question from the current interval. By definition $i$ is equal to or greater than 1 .

This formula reads like this: for every $d$ in the sequence $D$ select the ones, whose time values are greater than or equal to $(k-i) * a$, but less than $(k-i+1) * a$. $(k-i) \geq 0$ is used to ensure that we select a valid interval.

For the option \#2 the formula 1 will hold for the intervals $i_{k-1}$ and below, but for the interval $i_{k}$ it will change, so that:

$$
\begin{aligned}
F_{2}(D, T, i, a)= & i f(i>0) \\
& \text { then } F_{1} \\
& \text { else }[d: D \mid T-\Delta t \leq \text { time }(d) \leq T]
\end{aligned}
$$

where

$\Delta t$ is the time between $\mathrm{T}$ and the end of the previous interval. It can be computed as $T \bmod a$.

For the option \#3 for all the intervals the formula will change to:

$$
\begin{aligned}
& F_{3}(D, T, i, a)=[d: D \mid(\Delta t+(k-i-1) * a \leq \\
& \quad \text { time }(d)<\Delta t+(k-i) * a) \wedge(k-i-1) \geq 0]
\end{aligned}
$$

Each of $F_{1-3}$ would be preferred for different use cases. E.g. $F_{1}$ would be preferred for a case, when a user does not mind if an indicator is slightly lagging (up to the length $a$ of an interval) behind the current state of the market. $F_{2}$ would be preferred when a user is interested in a more up-to-date state of the market than what $F_{1}$ provides. $F_{3}$ would give the most accurate look of the market, but would require more computational resources.

In practice, option \#2 is the most common [10].

Using function $F_{2}(D, T, i, a)$ we can now define functions that help us calculate High, Low, Open, Close prices for the interval selected. Also it is important to understand that sometimes for rarely traded instruments we may receive no trades in a given time interval. We handle this situation by copying the High/Low/Open/Close price from the previous interval. If the previous interval has no prices, we continue traversing our sequence $D$ until we reach the start of our observation at time 0 in which case the resultant value is 0 .

Let us start with defining a generic function $I$, which searches for non-empty interval $i$ and applies an arbitrary function $X$ to the subsequence of values $d$, that fall within a selected interval. 


$$
\begin{array}{r}
I(D, T, i, a, X)=\text { if }\left(F_{2}(D, T, i, a)=\epsilon\right) \\
\text { then } \operatorname{if}(T-(\Delta t+(i+1) * a)>0) \\
\text { then }(I(D, T, i+1, a, X)) \\
\text { else } 0 \\
\text { else price }\left(X\left(F_{2}(D, T, i, a)\right)\right)
\end{array}
$$

where

$\epsilon$ is an empty sequence.

$X$ is an arbitrary function that takes a sequence as a parameter and returns an element of that sequence (e.g. head, which returns the first element).

The functions for computing High, Low, Open and Close can then be defined as follows:

High:

$$
H(D, T, i, a)=I\left(D, T, i, a, \max _{p}\right)
$$

where

$\max _{p}$ is the function returning an element of a sequence of 3-tuples with the highest price.

Low:

$$
L(D, T, i, a)=I\left(D, T, i, a, \min _{p}\right)
$$

where

$\min _{p}$ is the function returning an element of a sequence of 3-tuples with the lowest price.

Close:

$$
C(D, T, i, a)=I(D, T, i, a, \text { last })
$$

where

last is the function returning the last element of a sequence.

$$
O(D, T, i, a)=I(D, T, i, a, \text { head })
$$

where

head is the function returning the first element of a sequence.

Some indicators take into account the volume for a given interval. Unlike High/Low/Open/Close we do not need to retrieve the volume from any of the previous intervals if the current interval's volume is 0 .

$$
\operatorname{Vol}(D, T, i, a)=\sum_{j=0}^{\# F_{2}(D, T, i, a)-1} \operatorname{volume}\left(F_{2}(D, T, i, a) \cdot j\right)
$$

where

$j$ is used to select a member of a sequence, returned by $F_{2}$ function [11].

volume is used to select the volume member of a 3tuple as defined earlier in this section.

$\# F_{2}(D, T, i, a)$ is used to retrieve the length of a sequence.

\section{EWMA - Exponentially Weighted Moving Average}

Many indicators (including DMI indicator, which we discuss in the following sections) use EWMA. For the benefits of performance usually an EWMA is calculated according to this formula [19]:

$$
\begin{gathered}
\widehat{X}_{0, \alpha}=X_{0} \\
\widehat{X}_{k, \alpha}=(1-\alpha) * X_{k}+\alpha * \widehat{X}_{k-1, \alpha}
\end{gathered}
$$

where

$\widehat{X}_{k}$ is the value of exponentially smoothed price for the interval $k$.

$X_{k}$ is the price for the interval $k$.

$\alpha$ is a weighting parameter, which is chosen by the user. It needs to be in the range $0<\alpha \leq 1$.

From this formula one can see that we only need to store 1 previous value of EWMA to calculate the present value of EWMA.

Let us re-write the above formula for EWMA using our notation. First we need to calculate EWMA for the first period:

$$
E W M A_{X}(D, T, \mathrm{k}, a, \alpha)=X(D, T, \mathrm{k}, a)
$$

where

$\mathrm{k}$ is the number of the current interval, calculated as $\mathrm{k}=\lfloor T / a\rfloor$.

By using $\mathrm{k}$ as a value for $i$ we can refer to the first (index 0) interval of our observations. Thus in the above formula we set the EWMA value of a first interval to the value of function $X$ of that same interval.

Recall the meanings of the other variables:

$D$ is the sequence of 3-tuples, denoting time, price and volume of the trade.

$T$ is the current time.

$a$ is the length of an interval.

$X(D, T, i, a)$ (with $\mathrm{k}$ as a value for $i$ ) is used to denote $H(D, T, i, a), L(D, T, i, a), C(D, T, i, a)$, $O(D, T, i, a)$ or any other functions derived from these.

For every subsequent interval EWMA looks like follows.

$$
\begin{array}{r}
E W M A_{X}(D, T, i, a, \alpha)=(1-\alpha) * X(D, T, i, a)+ \\
\alpha * E W M A_{X}(D, T, i+1, a, \alpha)
\end{array}
$$

where

$i$ is the offset from the current interval.

\section{DMI indicator}

Let us now demonstrate our mathematical concepts in action by creating a rigorous model of Directional Movement Index (DMI) indicator. DMI is characterized as "a rather complex trend-following indicator" [9]. 
First, positive and negative directional movements are calculated - named $P D M$ and $N D M$ respectively. $P D M$ is current period's high minus previous period's high. $N D M$ is previous period's low minus current period's low.

$$
\begin{aligned}
& P D M_{\text {tran }}(D, T, i, a)=H(D, T, i, a)-H(D, T, i+1, a) \\
& N D M_{\text {tran }}(D, T, i, a)=L(D, T, i+1, a)-L(D, T, i, a)
\end{aligned}
$$

where

$i$ is an offset from current interval $k$, which is computed as $\mathrm{k}=\lfloor T / a\rfloor$.

tran stands for transitional and denotes transitional

$P D M$ and $N D M$ respectively, i.e. not the final ones.

$P D M$ is zero if $P D M_{\text {tran }}$ is negative, or less than $N D M_{t r a n}$. Otherwise it is equal to $P D M_{t r a n}$. The same logic applies to $N D M$.

$$
\begin{array}{r}
P D M(D, T, i, a)=i f\left(P D M_{\text {tran }}(D, T, i, a)<\right. \\
\left.N D M_{\text {tran }}(D, T, i, a)\right)
\end{array}
$$

then 0

$$
\text { else } \max \left(P D M_{t r a n}(D, T, i, a), 0\right)
$$

Likewise $N D M$ is:

$$
\begin{aligned}
& N D M(D, T, i, a)=i f\left(N D M_{\text {tran }}(D, T, i, a)<\right. \\
& \left.P D M_{\text {tran }}(D, T, i, a)\right) \\
& \text { else } \max \left(N D M_{\text {tran }}(D, T, i, a), 0\right)
\end{aligned}
$$

At the same time true range is calculated, which is the largest value of: current high minus current low, current high minus previous close or previous close minus current low.

$$
\begin{aligned}
T R(D, T, i, a)= & \max (H(D, T, i, a)-L(D, T, i, a), \\
& H(D, T, i, a)-C(D, T, i+1, a), \\
& C(D, T, i+1, a)-L(D, T, i, a))
\end{aligned}
$$

Then $P D M, N D M$ and $T R$ are smoothed with exponentially weighted moving average (EWMA).

Having smoothed $P D M, N D M$ and $T R$ we can now calculate positive and negative directional indices ( $P D I$ and $N D I$ respectively), which are calculated by division of smoothed $P D M$ by smoothed $T R$ for $P D I$ and by division of smoothed $N D M$ by smoothed $T R$ for $N D I$. The author of the indicator suggests that $\alpha$ should be set to $1 / 14$ or 0.07143 .

$$
\begin{aligned}
& P D I(D, T, i, a)=E W M A_{P D M}(D, T, i, a, 1 / 14) / \\
& E W M A_{T R}(D, T, i, a, 1 / 14) \\
& N D I(D, T, i, a)=E W M A_{N D M}(D, T, i, a, 1 / 14) / \\
& E W M A_{T R}(D, T, i, a, 1 / 14)
\end{aligned}
$$

Next directional movement $(D X)$ is defined as the the absolute difference between $P D I$ and $N D I$, divided by the sum of $P D I$ and NDI and the result multiplied by 100.

$$
\begin{aligned}
& D X(D, T, i, a)= \\
& 100 * a b s(P D I(D, T, i, a)-N D I(D, T, i, a)) / \\
& (P D I(D, T, i, a)+N D I(D, T, i, a))
\end{aligned}
$$

where

$a b s$ is the function returning an absolute value of a number.

Finally average directional movement $(A D X)$ is defined as an EWMA of $D X$ with $\alpha$ set to $1 / 14$ :

$$
A D X(D, T, i, a)=E W M A_{D X}(D, T, i, a, 1 / 14)
$$

\section{Implementation}

\subsection{Click Platform}

We have implemented DMI indicator described above on top of Click router. The Click Modular Router project [5, 1618] takes the approach of designing small objects which can be joined together to create a workflow or configuration, in an essentially arbitrary manner. Each object, known as a Click element, implements a processing function on a packet header at one of the layers of the OSI stack. The creation of Click configuration is facilitated by the Click language, that is a domain specific (DSL) language for building routing functions. Click router can run in kernel space as well as in user space, which should result in increased performance. From a development point of view an added benefit of Click router is that despite the integration with Linux kernel as a module it still allows code to be written in $\mathrm{C}++$. Click router encompasses an entire framework, which provides certain constructs, typically present in STL, but not available to kernel developer, such as hashmaps, strings, vectors etc.

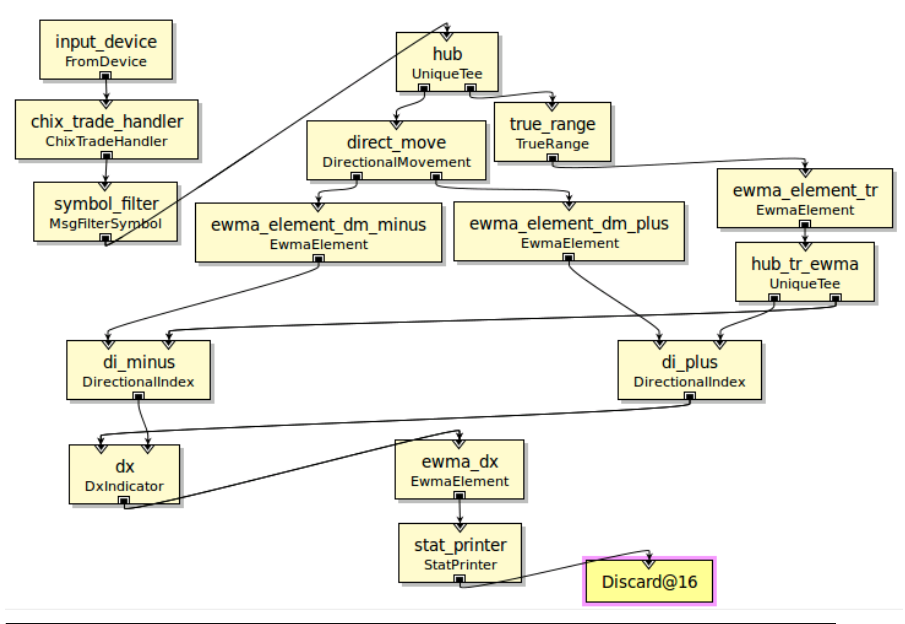

Figure 4. DMI indicator - topology. 
Another advantage is Click's multithreaded design (not used in our present tests), which allows to scale when deployed on many core CPUs.

The choice of Click platform for implementation is not binding. It can be any platform corresponding to a number of requirements. The next section expands a little on what makes Click a relatively good choice for this particular case.

\subsection{Mapping Maths to Click}

Our rigorous mathematical definition of a DMI indicator is essentially a cascade of interrelated functions, operating on a sequence of data (for most part). Click router has been designed specifically to process streams of data using a cascade of processing nodes (elements), which can be easily configured with the use of a DSL. Therefore the purpose of our experiments is to verify how well we can map the elements of our DSL onto the underlying platform (Click in this case, but it can be any suitable platform). We do the mapping manually. First we develop a set of Click elements (processing nodes), which correspond to functional constructs in our mathematical DSL. Second we link those elements together with the use of Click language to form a complete processing workflow - a DMI indicator. An overall picture of processing nodes and links/edges is shown in figure 4.

After processing the raw market data in chix_trade handler (to generate trades) and selecting the symbols of interest in symbol_filter we pass the trades to direct move, which calculates $P D M$ and $N D M$, and to true_range, which calculates $T R$. The output of these elements is smoothed using various instances of EwmaElement. di_minus and di_plus calculate $N D I$ and $P D I$ correspondingly. Together, the output of di_minus and di_plus is used to calculate $D X$ (in dx element). The output of $\mathrm{dx}$ is again smoothed using ewma_dx, which makes it $A D X$.

\subsection{Experimental Methodology}

Fidessa, the company sponsoring this paper, has provided the file with market data feed messages from Chi-X Europe stock exchange. This file contains about 1 hour worth of trading messages. The format of the messages corresponds to "CHIXMD FEED SPECIFICATION" [6]. In our experiments for simplicity purposes we have changed the transportation method of market data messages from TCP (as in original specification) to UDP, which is also widely used by very similar protocols, such as Multicast PITCH [1].

Since Linux kernel kernel does not immediately allow to do floating point calculations, we had to use fixed point arithmetic library to handle prices and other floating point calculations [2].

Click version 2.0.1 is used. Click package has been compiled for kernel space execution (as a kernel module) and for user space execution (as a standalone application). Linux kernel version 2.6.34.13 has been changed to enable the interception of packets. Two hooks were added to kernel netif_receive_skb function. The first hook is used by ti- mestamper kernel module, which attaches a time stamp (produced by rdtsc instruction) to a network packet of interest. The second hook is used only by Click in kernel mode to intercept the packet. Intel (R) Core (TM) i7 CPU 930 processor running at $2.8 \mathrm{GHz}$ was used for running the DMI indicator. This processor possesses an invariant time stamp counter [13], which allows to use it for accurate and predictable measurements. The latency was measured between the time stamp, produced by the timestamper kernel module, and the time stamp produced by StatPrinter element.

The rest of system settings were set to their default values as per standard Ubuntu distribution 12.04 and per generic Linux kernel version 2.6.34.13.

When Click is running in user space, it runs as a standalone application and simply consumes packets from a raw socket.

\subsection{Results}

A simple application was written, which sends UDP packets at a configurable message rate over computer network. Latency measurements were performed at 4 various rates: 10000, 50000, 100000 and 200000 messages per second. The results are shown in figure 5. Symbolfilter element was configured to filter trade messages for 2 of the most frequently traded symbols: BARCl and LLOYI. The latency measured is an average (mean) latency calculated over 6500 updates that reached StatPrinter. Standard deviation is calculated over the same 6500 messages as well. It is important to note that only around $2 \%$ of all market data contained in a file are trade messages.

The graphs present the results as candlesticks: the line in the middle is the average (mean) latency, the top and the bottom of the box are offset 0.5 of standard deviation from the mean. The maximum and the minimum values are represented by the top and bottom whiskerbars respectively. As one may see from the graphs, the in-kernel processing latency is significantly lower than that in userspace. We believe this is in part due to short processing path of DMI indicator relative to the processing path of network stack, which we bypass. The standard deviation in kernel space is also lower, meaning that much of the variability in execution time is caused by the network stack and OS/user space context switch. At 100000 messages per second the load on the system starts being excessive, leading to higher standard deviation. At 200000 messages per second the user level version of DMI indicator cannot keep up with data rates and only circa 2100 updates are processed, the rest are dropped. However the kernel space version of DMI indicator still manages to process all 6500 updates although at a cost of increased latency.

\section{Conclusion and Future work}

In this paper we have outlined the underpinning mathematical concepts for the rigorous construction of technical mar- 

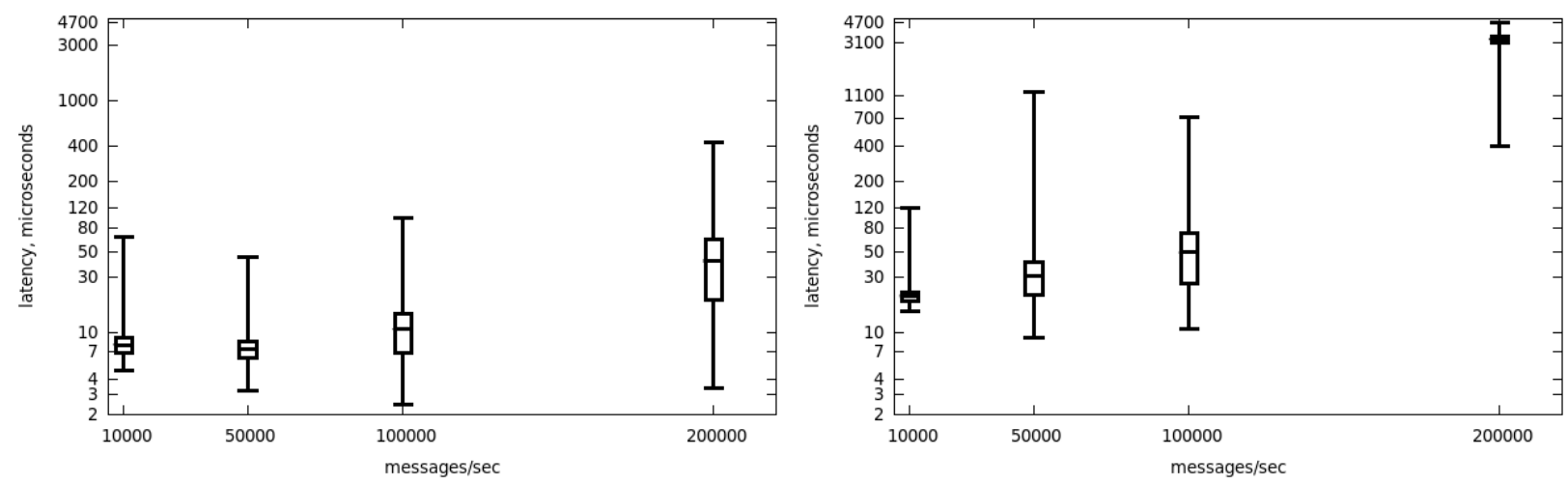

Figure 5. Execution time distribution of the DMI indicator in kernel space (left) and user space (right). The candlesticks indicate the minimum observed latency, half of a standard deviation below the mean, the mean observed latency, half of a standard deviation above the mean and the maximum latency. Latencies are depicted on a logarithmic scale.

ket indicators. These concepts are important because in a current environment there is no common notation for technical market indicators, neither there is any uniformity of notations. We hope that our paper will help to address both of these issues. Using mathematical concepts we have produced the model of a sample indicator: DMI indicator. We then produced an implementation of our sample indicator on top of Click router. The sequence data structure used in our model has mapped well onto the stream processing nature of Click router and the functional elements of DMI indicator have mapped well onto Click's concept of elements.

As expected, the latency of DMI indicator executing in kernel space is significantly lower than that in user space.

In the future we intend to expand our work to encompass running simultaneously a number of technical market indicators in a multi-threaded environment. We will concentrate on jointly optimising the Click routing functions for a set of indicators and instruments. We will also consider the processing of historical data as a computational problem, that allows a different set of code optimisations compared to real-time processing.

\section{Related Work}

Various online brokerage companies provide the software platforms for trading. These platforms often include the programming IDE and the language to allow users to create their own custom indicators. Sometimes the language in question is a general purpose imperative language, such as JavaScript in eSignal, C\# in Wealth-Lab, VB.NET in Stockfinder and others, but often it's a custom-made domain specific language (DSL), such as imperative EasyLanguage in Tradestation and Multicharts, the imperative AIQ EDS language in AIQ, imperative QScript in Wave59 and others. These DSLs are usually quite expressive and once again aimed at applying mathematical formulas to time series data. Quite often they are also tightly integrated with the trading platform, allowing the coding of execution strategies, alerts and other custom objects. The differences between DSLs make migra- tion from one platform to another not as easy and painless as it could be. One exception to this is EasyLanguage, which is used by both the TradeStation and MultiCharts.

FFTI [25] is an attempt to define a uniform notation for expressing technical market indicators. The authors take an approach whereby they define a number of aggregate functions, such as average, sum, product, min, max etc, and then use those functions with auxiliary parameters to define technical market indicators. They implement their concept as a web based tool. FFTI has little in common with our work as it uses a different approach, doesn't provide the same low level of underpinning mathematical concepts and does not intend to be modular and stream based in a way that our concept does.

Two books $[3,7]$ provide some of the most comprehensive overviews on the topic of technical market indicators, including their description, returns analysis and trading advice.

From time to time kernel space execution also receives its share of attention. Shukla et al [22] evaluate the performance of user space $\mu$ server and kernel space server TUX. Birch [4] evaluates the performance of an in-kernel packet capturing utility and Zander et al [24] compare the performance of an in-kernel UDP traffic generator and receiver vs. conventional tools such as tcpdump and CRUDE. All of them conclude that in certain (if not most) situations kernel space execution is superior to user space execution in terms of latency, performance and CPU load.

\section{Acknowledgments}

This work has been carried within the Northern Ireland Capital Markets Collaborative Network funded by a consortium of Queen's University Belfast, University of Ulster, Fidessa, NYSE Euronext, Citi, First Derivatives, Kofax and Invest Northern Ireland. The authors are grateful to Fidessa UK Ltd for providing the Chi-X data set which was used to conduct the experiments reported in this paper and for many useful 
discussions with Fidessa on this work. Konstantin Bakanov is supported by a $\mathrm{PhD}$ studentship from the Department of Employment and Learning NI. The research was carried out at the ECIT Institute at Queen's University Belfast.

The research leading to these results has received funding from the People Programme (Marie Curie Actions) of the European Union's Seventh Framework Programme (FP7/2007-2013) under the project NovoSoft, REA grant agreement no 327744 .

\section{References}

[1] BATS Chi-X Europe \| Support, . URL http://www. batstrading.co.uk/support/.

[2] Fixed Point Math Library for C | Free software downloads at SourceForge.net, . URL http://sourceforge.net/projects/fixedptc/.

[3] R. J. Bauer and J. R. Dahlquist. Technical Markets Indicators: Analysis \& Performance (Wiley Trading). John Wiley \& Sons, Inc., 605 Third Avenue, New York, NY 10158-0012, 1 edition, 1999.

[4] S. W. Birch. Performance characteristics of a Kernel-Space Packet Capture Module. Master's thesis, Dept. of Electrical and Computer Engineering, Air Force Institute of Technology, Air University, Wright-Patterson Air Force Base, OH, 2010.

[5] B. Chen and R. Morris. Flexible control of parallelism in a multiprocessor pc router. In Proceedings of the 2001 USENIX Annual Technical Conference (USENIX '01), pages 333-346, Boston, Massachusetts, June 2001.

[6] Chi-X Europe Limited. CHIXMD Feed Specification. Doc Revision: 1.6. Historical specification, may be available from BATS Trading Limited: http://www.batstrading.co.uk/, June 2010.

[7] R. W. Colby. The Encyclopedia Of Technical Market Indicators, Second Edition. McGraw-Hill, Two Penn Plaza, New York, USA, 2 edition, 2003.

[8] R. W. Colby. Types of Technical Market Indicators: Trend, Momentum, Sentiment. In The Encyclopedia Of Technical Market Indicators, Second Edition, chapter 1, pages 7-8. McGraw-Hill, Two Penn Plaza, New York, USA, 2 edition, 2003.

[9] R. W. Colby. Directional Movement Index (DMI). In The Encyclopedia Of Technical Market Indicators, Second Edition, pages 212-213. McGraw-Hill, Two Penn Plaza, New York, USA, 2 edition, 2003.

[10] Fidessa Group Plc. private communication.

[11] D. Gries and F. B. Schneider. A theory of sequences. In $A$ Logical Approach to Discrete Math, chapter 13, pages 251264. Springer-Verlag New York Inc., 185 Fifth Avenue, New York, NY 10010, USA, 1993.

[12] C. D. K. II and J. R. Dahlquist. The Basic Principle of Technical Analysis - The Trend. In Technical Analysis: The Complete Resource for Financial Market Technicians (2nd Edition), chapter 2, pages 9-11. FT Press, Upper Saddle River, New Jersey, USA, 2 edition, July 2011. ISBN 9780137059447.

[13] Intel Corporation. Invariant TSC. In Intel 64® and IA-32 Architectures Software Developer's Manual Volume 3 (3A \& 3B): System Programming Guide, chapter 16.12.1, pages 16-
50. Intel, 2011.

[14] B. Johnson. Institutional trading types. In Algorithmic Trading and DMA: An introduction to direct access trading strategies, chapter 1.4, pages 8-10. 4Myeloma Press, February 2010.

[15] E. Kohler. The Click Modular Router. PhD thesis, Dept. of Electrical Engineering and Computer Science, Massachusetts Institute of Technology, Cambridge, MA, 2001.

[16] E. Kohler. Click for Measurement. Technical Report TR060010, UCLA Computer Science Department, 405 Hilgard Ave Los Angeles, CA 90095, United States, February 2006.

[17] E. Kohler, R. Morris, and B. Chen. Programming language optimizations for modular router configurations. SIGARCH Computer Architecture News, 30 (5):251-263, Oct. 2002. ISSN 0163-5964. . URL http://doi . acm.org/10.1145/635506.605424.

[18] E. Kohler, R. Morris, and M. Poletto. Modular components for network address translation. In Open Architectures and Network Programming Proceedings, 2002 IEEE, pages 3950,2002 . .

[19] J. Loveless, S. Stoikov, and R. Waeber. Online algorithms in high-frequency trading. Commun. ACM, 56 (10):50-56, Oct. 2013. ISSN 0001-0782. . URL http://doi.acm.org/10.1145/2507771.2507780.

[20] M. Resta. Towards an artificial technical analysis of financial markets. In Proceedings of the IEEE-INNS-ENNS International Joint Conference on Neural Networks, 2000. IJCNN 2000, volume 5, pages 117-122, 2000. .

[21] A. Rodriguez-Gonzalez, F. Guldris-Iglesias, R. ColomoPalacios, G. Alor-Hernandez, and R. Posada-Gomez. Improving $\mathrm{N}$ calculation of the RSI financial indicator using neural networks. In 2010 2nd IEEE International Conference on Information and Financial Engineering (ICIFE), pages 49-53, 2010. .

[22] A. Shukla, L. Li, A. Subramanian, P. A. S. Ward, and T. Brecht. Evaluating the performance of user-space and kernel-space web servers. In Proceedings of the 2004 conference of the Centre for Advanced Studies on Collaborative research, pages 1-13, 2004.

[23] M. Tanaka-Yamawaki and S. Tokuoka. Adaptive use of technical indicators for the prediction of intra-day stock prices. Physica A: Statistical Mechanics and its Applications, 383(1): 125 - 133, 2007. .

[24] S. Zander, D. Kennedy, and G. Armitage. KUTE A High Performance Kernel-based UDP Traffic Engine. Technical Report 050118A, Centre for Advanced Internet Architectures, Swinburne University of Technology, Melbourne, Australia, 2005.

[25] A. Zubayer, M. Musharraf, and R. Ahmed. FFTI: Free Form Technical Indicator. In 2011 3rd International Conference on Computer Research and Development (ICCRD), volume 1, pages 87-91, 2011. . 